\section{Janvier 1995}

\section{XVlème Cours de Chirurgie de la Main et du Membre Supérieur de l'Hôpital Bichat (Paris)}

\section{Paris, 26-27 janvier 1995}

Organisé par J.Y. Alnot, C. Oberlin, R. Bleton

Thèmes:

- Pathologies de l'épaule. L'épaule neurologique et paralytique et les traumatismes récents.

- Pathologies traumatiques et non traumatiques du poignet et de la pronosupination

- Actualités chirurgicales

Renseignements et inscriptions: Madame Copin, Secrétaire du Pr J.Y. Alnot Hôpital Bichat, 46, rue Henri Huchard, F-75877 Paris Cedex 18, Tél. 40257501

\section{Avril 1995}

\section{8ème Journées Lyonnaises de Chirurgie du Genou}

\section{Lyon, 6-8 avril 1995}

H. Dejour, J.P. Carret, Ph. Neyret

Centre Hospitalier Lyon-Sud

25 ans de recherches, d'études, d'essais, de résultats... Des lésions ligamentaires, rotuliennes, fémoro-tibiales. La chirurgie prothétique

Renseignements et inscriptions: Nataly Revel, Service du Professeur Dejour, Centre Hospitalier Lyon-Sud, F-69310 Pierre Bénite, Tél. 788614 26, Fax 72399642

\title{
Analyse de livre
}

\section{Chirurgie du membre supérieur - SOFCOT}

Une sélection des conférences d'enseignement. (1993) Expansion Scientifique Française, Paris, 179 p, nombreuses illustrations, Frs. 260

Cet ouvrage est constitué des conférences d'enseignement de la SO.F.C.O.T. partes de 1987 à 1992 concernant les pathologies du membre supérieur (de l'épaule à la main en passant par le coude et le poignet).

Ainsi, D. Patte donne son avis à partir de son expérience personnelle sur les luxations traumatiques des articulations acromio et sterno claviculaires. L. Sedel présente de manière condensée les possibilités de traitement des paralysies des différents muscles de l'épaule. C. Gerber nous présente son expérience des instabilités de l'épanle.

A. Tanguy puis $\mathrm{H}$. Bracq apportent l'experience de chirurgiens orthopédistes pédiatriques à propos des fractures des deux os de l'avant bras et des fractures supracondyliennes de l'enfant, dont la grande fréquence intéresse tous les chirurgiens.

M.B. Wood rapporte son expérience de la restauration de la flexion du coude, qui est un geste capital de la chirurgie réparatrice des paralysies du plexus brachial.

C. Oberlin nous rappelle (ou nous explique) les bases et implications cliniques des principales instabilités du poignet. J.Y. Alnot puis J.L. Condamine nous présentent à partir des bases biomécaniques du poignet et des articulations métacarpo phalangiennes et interphalangiennes proximales l'arthroplastie idéale et celles actuellement proposées.

F. Iselin présente de manière simple et didactique le traitement des lésions traumatiques de l'appareil extenseur. A. Gilbert fait le point sur les progrès récents du traitement de quelques malformations congénitales et planifie celui ci.

A. O. Narakas présente à propos de son importante expérience les syndromes canalaires (fréquents ou moins fréquents) du membre supérieur.

Cet ouvrage intéressera tous les chirurgiens jeunes ou moins jeunes intéressés par la chirurgie de la main et du membre supérieur. Ceci leur permettra de se faire une idée de sujets peu fréquents et sera une base de départ pour les approfondir, ou apportera, pour des pathologies pouvant paraître compliquées au premier abord, des lignes de traitement simples. 